\title{
Feasibility of Rehabilitation Training With a Newly Developed, Portable, Gait Assistive Robot for Balance Function in Hemiplegic Patients
}

\author{
Junhyun Sung, MD' ${ }^{1}$, Sehoon Choi, $\mathrm{MD}^{1}$, Hyunbae Kim, $\mathrm{MD}^{2}$, Gyuhan Lee, $\mathrm{MD}^{2}$, \\ Changsoo Han, $\mathrm{PhD}^{3}$, Younghoon Ji, $\mathrm{MS}^{3}$, Dongbin Shin, $\mathrm{MS}^{3}$, Seunghoon Hwang, $\mathrm{MS}^{3}$, \\ Deokwon Yun, $\mathrm{PhD}^{3}$, Hyeyoun Jang, $\mathrm{PhD}^{3}$, Mi Jung Kim, $\mathrm{MD}, \mathrm{PhD}^{1}$ \\ ${ }^{1}$ Department of Rehabilitation Medicine, Hanyang University College of Medicine, Seoul; \\ ${ }^{2}$ Department of Rehabilitation Medicine, Rusk Bundang Rehabilitation Hospital, Seongnam; \\ ${ }^{3}$ Department of Robot Engineering, CIM \& Robotics Laboratory, Hanyang University, Ansan, Korea
}

\begin{abstract}
Objective To investigate the clinical feasibility of a newly developed, portable, gait assistive robot (WA-H, 'walking assist for hemiplegia') for improving the balance function of patients with stroke-induced hemiplegia.

Methods Thirteen patients underwent 12 weeks of gait training on the treadmill while wearing WA-H for 30 minutes per day, 4 days a week. Patients' balance function was evaluated by the Berg Balance Scale (BBS), FuglMeyer Assessment Scale (FMAS), Timed Up and Go Test (TUGT), and Short Physical Performance Battery (SPPB) before and after 6 and 12 weeks of training.

Results There were no serious complications or clinical difficulties during gait training with WA-H. In three categories of BBS, TUGT, and the balance scale of SPPB, there was a statistically significant improvement at the 6th week and 12th week of gait training with WA-H. In the subscale of balance function of FMAS, there was statistically significant improvement only at the 12th week.

Conclusion Gait training using WA-H demonstrated a beneficial effect on balance function in patients with hemiplegia without a safety issue.
\end{abstract}

Keywords Robotics, Stroke, Hemiplegia, Gait, Rehabilitation

Received June 20, 2016; Accepted September 7, 2016

Corresponding author: Mi Jung Kim

Department of Rehabilitation Medicine, Hanyang University College of Medicine, 222-1 Wangsimni-ro, Seongdong-gu, Seoul 04763, Korea. Tel: +82-22290-9353, Fax: +82-2-2282-0772, E-mail: kimmjreh@hanyang.ac.kr

ORCID: Junhyun Sung (http://orcid.org/0000-0002-7913-1614); Sehoon Choi (http://orcid.org/0000-0003-2840-9783); Hyunbae Kim (http://orcid. org/0000-0002-7209-4574); Gyuhan Lee (http://orcid.org/0000-0002-6188-460X); Changsoo Han (http://orcid.org/0000-0002-8561-847X); Younghoon Ji (http://orcid.org/0000-0003-0897-6211); Dongbin Shin (http://orcid.org/0000-0001-5702-5557); Seunghoon Hwang (http://orcid.org/0000-00021856-0829); Deokwon Yun (http://orcid.org/0000-0001-9993-7933); Hyeyoun Jang (http://orcid.org/0000-0002-5012-9860); Mi Jung Kim (http://orcid. org/0000-0003-2920-9900).

@ This is an open-access article distributed under the terms of the Creative Commons Attribution Non-Commercial License (http://creativecommons.org/ licenses/by-nc/4.0) which permits unrestricted noncommercial use, distribution, and reproduction in any medium, provided the original work is properly cited. Copyright $\odot 2017$ by Korean Academy of Rehabilitation Medicine 


\section{INTRODUCTION}

Gait disturbance is one of the major complications in stroke patients and limits the patient's activities of daily living. An inability or an impaired ability to walk is a significant contributor to long-term disability and burden of care after stroke, and improved walking function is an important factor in returning to social life [1]. Because standing and walking balance are important predictors of the safe performance of gait and other functional activities, an extensive range of programs has been employed to improve standing and walking balance of hemiplegic patients. These include muscle-strengthening exercises, gait exercises, balance exercises, aerobic exercises, patient education and physical therapy [2]. Assistive robotic devices are a newly introduced rehabilitative approach for gait disturbance, and they have received considerable attention as a changing paradigm from classical rehabilitative training [3]. The potentially positive benefit of robotic gait training is that it involves repeatedly undergoing sufficient and accurate training for a prolonged period without laborious intervention of physical therapists. Lokomat (Hocoma AG, Zurich, Switzerland) is the first robotic-driven gait orthosis with electromechanical drives to assist the walking movements of gait-impaired patients on a treadmill by supporting the body weight $[4,5]$. Like Lokomat, a conventionally developed, gait assistive, exoskeletal robot is huge in size and utilized only with treadmill walking with a suspension system [3]. One disadvantage of treadmill training using Lokomat or similar devices is the effort needed by therapists to move patients on or off the treadmill, set the paretic limbs, and apply the suspension system [6]. In addition, the fixed speed of the treadmill can limit the therapy intensity. These factors make the exoskeletons less portable and less practical, especially for paralyzed patients. The cost of the device is another important issue. Most exoskeleton systems currently available may be priced as high as US $\$ 100,000$ or more, which is not cost-effective, and only affordable to a small number of people [7]. To solve the above mentioned problems and to assist the gait of patients with hemiplegia, we have developed a wearable and portable, light weight, gait assistive robot named WA-H ('walking assist for hemiplegia') that can be adapted directly for use at home and rehabilitation centers [8]. Compared to other gait assistive robots, such as Lokomat, presently used in the medical field, this light weight, gait assistive, robot system can be used without a treadmill, is portable for outdoor activities, and can be implemented at a relatively lower cost than integrated systems. Therefore it can provide gait training in various environments such as home and/or outdoors, depending on the condition of the patient. This study aims to investigate the clinical feasibility of the portable, wearable gait assistive robot, WA-H, on the balance function of patients with stroke-induced hemiplegia. This is a second study following up on a pilot study conducted in 2013 with 2 stroke patients to confirm the device stability [9]. Accordingly, this study is designed to confirm further the efficacy of the device with a greater number of patients. The main hypothesis is that gait training with a newly developed, portable, gait assistive robot would be superior to previous devices with respect to the restoration of gait ability and balance. The clinical efficacy of the newly developed, gait assistive robot for improving the gait ability and balance function of stroke patients undergoing rehabilitation was evaluated.

\section{MATERIALS AND METHODS}

\section{Subject selection}

The study protocol was approved by the Institutional Review Board of Hanyang University. The clinical intervention was conducted at the RUSK Bundang Hospital, in Gyeonggi Province, Korea from January to August 2015. The pre- and post-assessment was conducted in the Hanyang University Medical Center and related facilities. All the patients were hospitalized, and a classical comprehensive rehabilitation program had been done before this study. The patients were informed about the aim and design of this study by medical doctors, and they subsequently provided the signed consent.

The inclusion criteria were as follows: (1) hemiplegic patient with stroke, based on history and medical record; (2) patient who was neurologically and medically stable; (3) patient who could walk independently but showed gait disturbance (limping, foot drop, etc.); and (4) patient capable of gait training for 30 minutes or more.

The exclusion criteria were the following: (1) patient with other causes of hemiplegia except stroke, such as traumatic brain injury, brain tumor, or seizure; (2) patient with incompatible medical conditions for gait train- 
ing; (3) patient with severely impaired conversation or cognition; (4) patient with other uncontrollable medical or surgical diseases; and (5) patient considered inappropriate to the study by researchers. According to the above criteria 13 hemiplegic patients (9 men and 4 women, 9 with ischemic stroke and 4 with hemorrhagic stroke) with stroke were recruited.

The mean age of the patients was 56.6 years and the mean duration since stroke onset was 12.7 months. Eleven patients were able to ambulate under supervision without any kinds of braces or supports; one patient could walk with a single cane, and the other with an ankle foot orthosis. Gait speed was measured by walking back-and-forth in a 20-m corridor for 6 minutes. Compared with the study of Oberg et al. [10], all the participants exhibited a diminished speed compared to the normal range of healthy people of the same age. Four patients had a speed lower than $25 \%$ of the normal range, 6 patients showed a speed $25 \%$ to $50 \%$ of the normal range, and 3 patients showed a speed $50 \%$ to $75 \%$ of the normal range of healthy people of the same age. All the participants showed toe dragging and circumduction gait during the swing phase, and decreased stance time on the paretic side due to motor weakness. All could walk independently with or without assistive devices despite an unstable and slow manner (Table 1).

\section{Newly developed, portable, gait assistive robot}

The newly developed, portable, gait assistive robot (WA$\mathrm{H})$ was used in this study. The specifications of WA-H are as follows: the weight of the system including battery is $25 \mathrm{~kg}$; the dimensions are $595 \mathrm{~mm} \times 390 \mathrm{~mm} \times 1300 \mathrm{~mm}$; maximum operating time is one hour; maximum torque of hip joint is $188 \mathrm{Nm}$ and knee joint is $55.5 \mathrm{Nm}$; the range of walking speed is from $0.5 \mathrm{~km} / \mathrm{h}$ to $3 \mathrm{~km} / \mathrm{h}$. The main movement of the gait assistive robot is achieved in the sagittal plane during gait training. Thus, it uses motors to assist the movement of the knee and hip joints of the wearer in the sagittal plane. Additionally, in the coronal plane, passive hip joints were used to make a tracing of the wearer's joint movement for weight shifting during walking, and were designed to adjust and fit the position of the joints. Ankle joints were designed to prevent foot drop and assist ankle dorsiflexion by using a spring attachment. All joints were limited not to exceed the normal range of motion. The affected and sound sides were controlled independently with different methods. The sound side was controlled by compensating friction in each driving unit so that the robot would not disturb the movement of the user. Also, an assist algorithm was

Table 1. Demographic data of each participant

\begin{tabular}{ccccclccc}
\hline $\begin{array}{c}\text { Patient } \\
\text { no. }\end{array}$ & Sex & $\begin{array}{c}\text { Weight } \\
(\mathbf{k g})\end{array}$ & $\begin{array}{c}\text { Height } \\
(\mathbf{c m})\end{array}$ & $\begin{array}{c}\text { Age } \\
(\mathbf{y r})\end{array}$ & \multicolumn{1}{c}{ Diagnosis } & $\begin{array}{c}\text { Duration } \\
\text { since disease } \\
(\mathbf{m o})\end{array}$ & $\begin{array}{c}\text { Assistive } \\
\text { device }\end{array}$ & $\begin{array}{c}\text { Gait speed } \\
(\mathbf{c m} / \mathbf{s})\end{array}$ \\
\hline 1 & M & 65 & 170 & 54 & Left BG ICH & 17 & No & 58.8 \\
\hline 2 & M & 68 & 174 & 74 & Left BG \& CR infarction & 20 & No & 33.3 \\
\hline 3 & F & 57 & 156 & 50 & Left BG \& CR infarction & 12 & No & 19.4 \\
\hline 4 & M & 75 & 160 & 45 & Left BG ICH & 5 & No & 46.4 \\
\hline 5 & M & 61 & 160 & 45 & Left BG infarction & 13 & Monocane & 22.2 \\
\hline 6 & M & 78 & 175 & 58 & Left MCA infarction & 12 & No & 44.4 \\
\hline 7 & M & 70 & 172 & 68 & Left MCA infarction & 12 & AFO & 15.6 \\
\hline 8 & M & 91 & 176 & 47 & Rig ht BG ICH \& both IVH & 12 & No & 94.7 \\
\hline 9 & F & 60 & 157 & 66 & Left medulla \& right & 15 & No & 37.8 \\
\hline 10 & F & 60 & 150 & 64 & Left BG infarction & 10 & No & 67.2 \\
\hline 11 & M & 59 & 164 & 69 & Left thalamus ICH & 20 & No & 22.8 \\
\hline 12 & M & 64 & 164 & 38 & Right MCA infarction & 4 & No & 60.3 \\
\hline 13 & F & 55 & 160 & 58 & Left BG \& CR infarction & 13 & No & 55.6 \\
\hline
\end{tabular}

$\mathrm{BG}$, basal ganglia; ICH, intracranial hemorrhage; $\mathrm{CR}$, corona radiata; $\mathrm{MCA}$, middle cerebral artery; IVH, intraventricular hemorrhage; AFO, ankle foot orthosis. 
established to aid the gait of the user. The affected side was controlled to follow the gait trajectory which was entered by the sound side and could be modified by operators. The duration of the target trajectory was designed to adjust according to gait speed. The target trajectory was created by the normal side's stride, gait cycle and gait pattern. These were measured by FSR (force sensitive resistor) sensors in the insole and an IMU (inertial measurement unit) sensor in the pelvic area composed of a 3 -axis acceleration sensor and a 2-axis gyro sensor (Fig. 1). Two FSR sensors in the insole measured heel strike, heel off, and toe off in the sound leg, detecting contact between heel and toe to the ground [9]. The IMU sensor in the pelvic area detected the patient's movement and converted signals into movements of roll and pitch axis through the Kalman filter. All data such as step time, joint angles and gait speed were entered into the main controller unit (MCU) and the target trajectory was created. The target trajectory was played via four motors in the

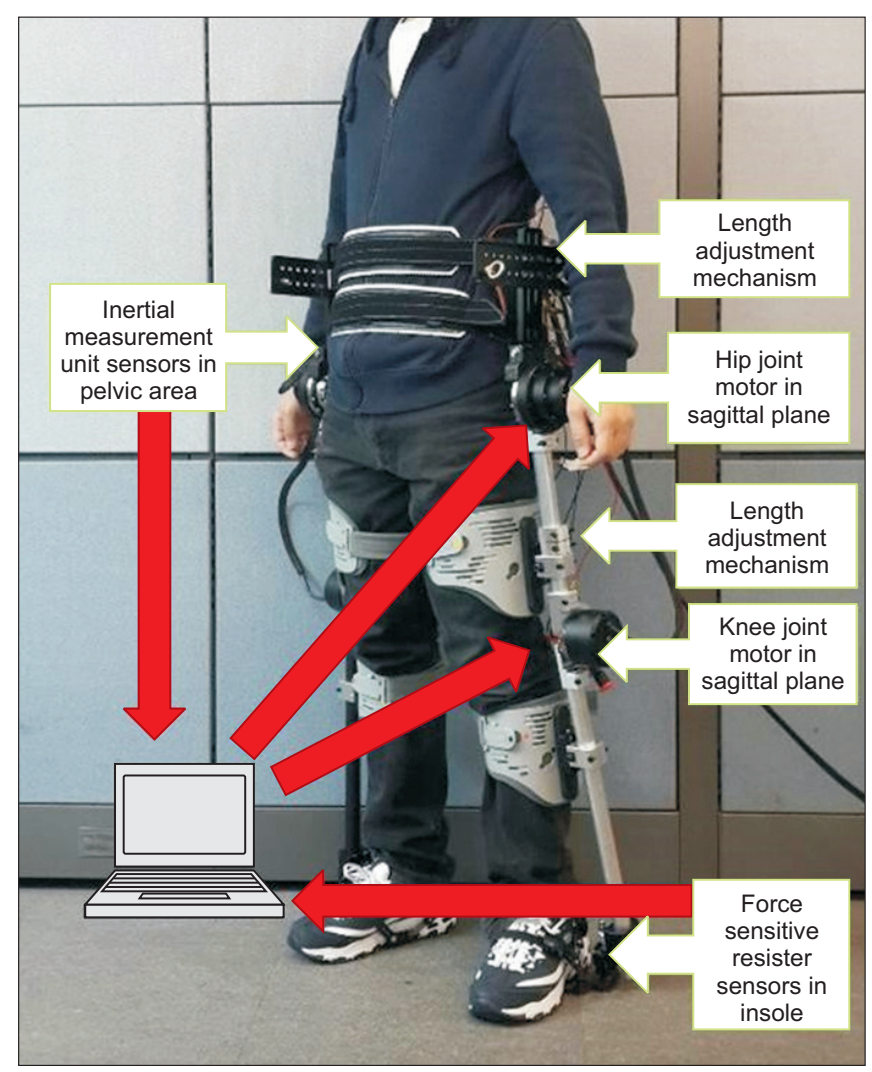

Fig. 1. WA-H (walking assist for hemiplegia) system. Target trajectory was created by normal side's sensors, and motors at knee and hip joints were used to assist the movement. hip and knee joints in the affected side once heel strike occurred in the sound side. A personal computer was connected to the MCU with a wireless local area network. Operators can control and monitor the training, and save the training data using the personal computer. WA-H is relatively lighter than existing embedded devices such as Lokomat and is designed to be equipped and used in any location without requiring additional devices, all of which contribute to its practicality [8].

\section{Training program}

Thirteen patients underwent 12 weeks of gait training while wearing WA-H on the treadmill for 30 minutes per day, 4 times a week. To prevent injury, patients walked with a hand rail and suspension harness support, which was not used to support patient's weight but to prevent falling and emergency situations (Fig. 2). Conventional rehabilitation training such as occupational therapies that enhance upper limb function, and other rehabilitation therapies that have no direct correlation to gait or lower limb training such as speech therapy, were provided without restriction. The rehabilitation program that employed the wearable assistive robot on the patient's affected limb was as follows: the subjects put on the device and had a familiarization session to become familiar with the experimental conditions for 5 minutes; the duration of gait training was 30 minutes in each session, and the training was conducted 4 times a week for 12 weeks. During the first week, training was conducted, not on the treadmill, but on the floor with the safety lift for adapting to the equipment and for safety training. After that, training on the treadmill was started. The basic training protocol is described in the following sentences, but it could

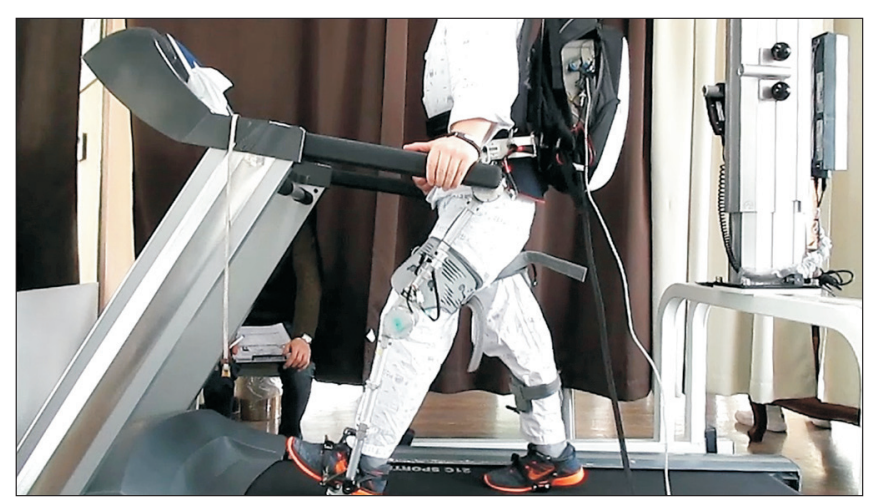

Fig. 2. Gait training with WA-H on the treadmill with hand rail. 
be changed flexibly according to the general condition and function of the patients. During the first 5 minutes, training was conducted at a speed of $1.5 \mathrm{~km} / \mathrm{h}$. During the next 20 minutes, treadmill speed was increased to 2.0 $\mathrm{km} / \mathrm{h}$, and for the last 5 minutes, treadmill speed was decreased to $1.5 \mathrm{~km} / \mathrm{h}$. From the 7th to 12 th week of training, the maximum speed was regulated up to $2.5 \mathrm{~km} / \mathrm{h}$ [9]. Evaluations were conducted before the training began, and conducted in the 6th week, and in the 12th week to test and compare the improvement of lower limb balance after using the gait assistive robot.

\section{Outcome measures}

Patients' balance was evaluated by the Berg Balance Scale (BBS), Fugl-Meyer Assessment Scale (FMAS), Timed Up and Go Test (TUGT), and Short Physical Performance Battery (SPPB) before, and after 6 and 12 weeks of training by two medical doctors with supervision by two engineers.

\section{Timed Up and Go Test}

TUGT assesses basic mobility and reflects the ability to transfer from sitting to standing and to walk a short distance. Subjects were required to stand up from a chair with armrests, walk $3 \mathrm{~m}$, turn around, return to the chair, and sit down using their customary walking aids. The best time, measured in seconds, from three trials was taken for analysis. The test is a reliable and valid instrument for stroke patients [11].

\section{Balance item of Short Physical Performance Battery}

SPPB is a performance-based test of lower extremity function designed for elderly participants. In the balance test, the participant holds his/her balance for 10 seconds in three standing positions with eyes open: feet side by side, feet in semi-tandem stance (big toe of one foot touching heel of other foot), and feet in tandem stance (heel to toe). One point each is given when feet side by side or semi tandem stance can be sustained for at least 10 seconds, 1 point when tandem stance is sustained for at least 3 seconds, and 2 points when tandem stance is sustained for 10 seconds, with 4 points as total [12].

\section{Berg Balance Test}

BBS, consisting of 14 tasks, was developed to measure balance among older people with impairment in bal- ance function by assessing the performance of functional tasks. Each task is scored on a scale ranging from 0 to 4 points, and the total score is used as the index of balance ability [13].

\section{Subscale of balance function of Fugl-Meyer Assessment Scale}

FMAS is a 226-point multi-item Likert-type scale developed as an evaluative measure of recovery from hemiplegic stroke. It is divided into five domains: motor function, sensory function, balance, joint range of motion, and joint pain. Each domain contains multiple items, each scored on a 3-point ordinal scale ( 0 , cannot perform; 1 , performs partially; 2, performs fully). The balance domain consists of 7 items and 14 points for sitting and standing balance [14].

\section{Statistical analysis}

All parametric data were expressed as mean \pm standard deviation. The Wilcoxon signed-rank test and the Friedman test were used to evaluate differences between the baseline measurements and outcomes after the gait training with the newly developed, gait assistive robot. Spearman correlation coefficients were used to assess the relationship among changes in outcome measures. A p-value less than 0.05 was considered statistically significant. Data analyses were performed using the SPSS program ver. 18.0 for Windows (SPSS Inc., Chicago, IL, USA).

\section{RESULTS}

The experimental group of 13 subjects was trained with the gait assistive robot for 12 weeks according to training program described above. Two engineers participated in the experiment to adjust the appropriate gait speed and prepare for unexpected safety accidents such as falling. Four different measurements of balance were taken prior to training (baseline), and after the 6th week and 12th week of training.

\section{Berg Balance Scale}

There was an increase in BBS after 12 weeks training with the gait assistive robot (Table 2). There were statistically significant improvements at the 6th week and 12th week. An increase in the BBS was observed in 12 of the 13 patients, and 1 patient had the same score as prior to 
training (Fig. 3).

\section{Timed Up and Go Test score}

All 13 patients showed decreased TUGT time after 12 weeks of training with the gait assistive robot (Table 3, Fig. 4). There were also statistically significant improvements in scores at the 6 th week as well as at the 12th week.

\section{Balance item of Short Physical Performance Battery}

Eight patients had an increase in the balance item of SPPB after 12 weeks of training with the gait assistive robot, and 5 patients' scores were unchanged (Table 4, Fig. 5). The improvement in scales was statistically significant at both the 6 th week and the 12 th week.

\section{Subscale of balance function of Fugl-Meyer Assessment Scale}

Nine patients had an increase in the subscale of balance function of FMAS after 12 weeks of training with the gait assistive robot, and 4 patients' scores were unchanged (Table 5, Fig. 6). Improved scores were seen at the 6th

Table 2. Changes in the BBS in baseline, the 6th and 12th week $(\mathrm{n}=13)$

\begin{tabular}{lcc}
\hline & BBS & p-value \\
\hline Baseline & $43.6 \pm 5.8$ & \\
6th week & $46.5 \pm 5.2$ & $0.005^{*}$ \\
12th week (end of training) & $48.2 \pm 4.8$ & $0.002^{*}$ \\
\hline
\end{tabular}

Values are presented as mean \pm standard deviation. BBS, Berg Balance Scale. ${ }^{*} \mathrm{p}<0.05$ vs. baseline.

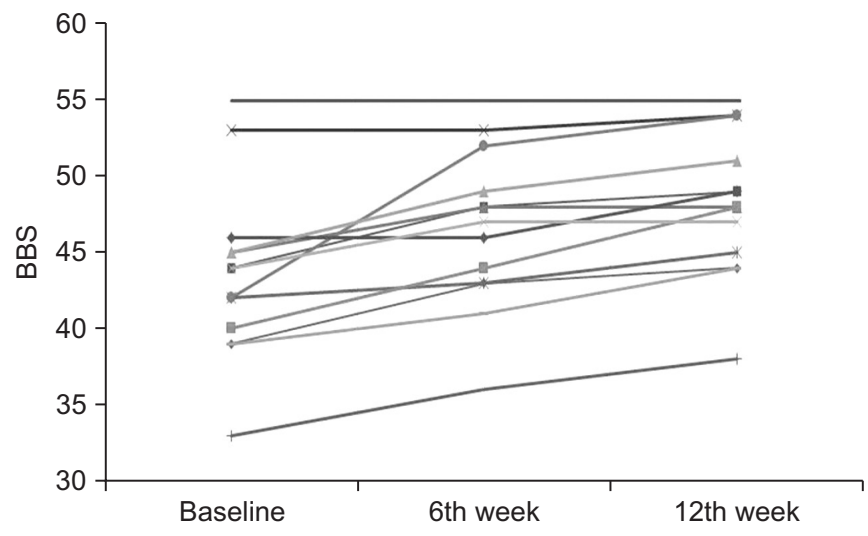

Fig. 3. Changes in the Berg Balance Scale (BBS) for 13 patients in baseline, the 6th week and 12th week. week and 12th week, but there was statistical significance only at the 12th week. The Friedman test showed that all scales were improved significantly after 6 weeks and 12 weeks of training $(\mathrm{p}<0.05)$. There were no distinctive correlations among changes in outcome measures.

\section{Adverse event during training}

In the 3rd week of training, one subject (case 8) exhibited a seizure like motion of 6 minutes duration 3 hours after the morning training, and was sent directly to the emergency room for evaluation. The subject showed no abnormal findings in blood chemistry tests. Further evaluations like electroencephalogram (EEG) were suggested, but were refused by the patient due to three previous histories of seizures. The subject showed no abnormal clinical symptoms during 2 hours in the emergency room and was discharged after all symptoms subsided. The subject showed no other symptoms afterwards, and continued with training because the rehabilitation program using the gait assistive robot was considered to have little correlation to seizures. No further seizure like motion

Table 3. Changes in the TUGT score in baseline, the 6th and 12 th week $(\mathrm{n}=13)$

\begin{tabular}{llc}
\hline & TUGT (s) & p-value \\
\hline Baseline & $25.8 \pm 12.0$ & \\
6th week & $21.6 \pm 9.4$ & $0.003^{*}$ \\
12th week (end of training) & $19.7 \pm 8.9$ & $0.001^{*}$ \\
\hline
\end{tabular}

Values are presented as mean \pm standard deviation. TUGT, Timed Up and Go Test. ${ }^{*} \mathrm{p}<0.05$ vs. baseline.

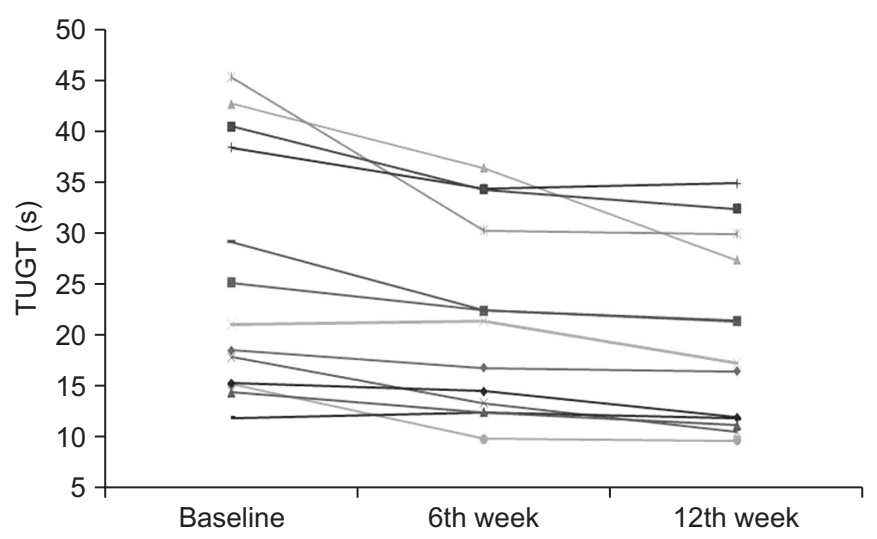

Fig. 4. Changes in the Timed Up and Go Test (TUGT) score for 13 patients in baseline, the 6th week and 12th week. 
Table 4. Changes in the B-SPPB in baseline, the 6 th and 12 th week $(n=13)$

\begin{tabular}{lcc}
\hline & B-SPPB & p-value \\
\hline Baseline & $2.5 \pm 1.2$ & \\
6th week & $3.0 \pm 0.9$ & $0.020^{*}$ \\
12th week (end of training) & $3.3 \pm 0.8$ & $0.007^{*}$ \\
\hline
\end{tabular}

Values are presented as mean \pm standard deviation. B-SPPB, balance item of Short Physical Performance Battery.

${ }^{*} \mathrm{p}<0.05$ vs. baseline.

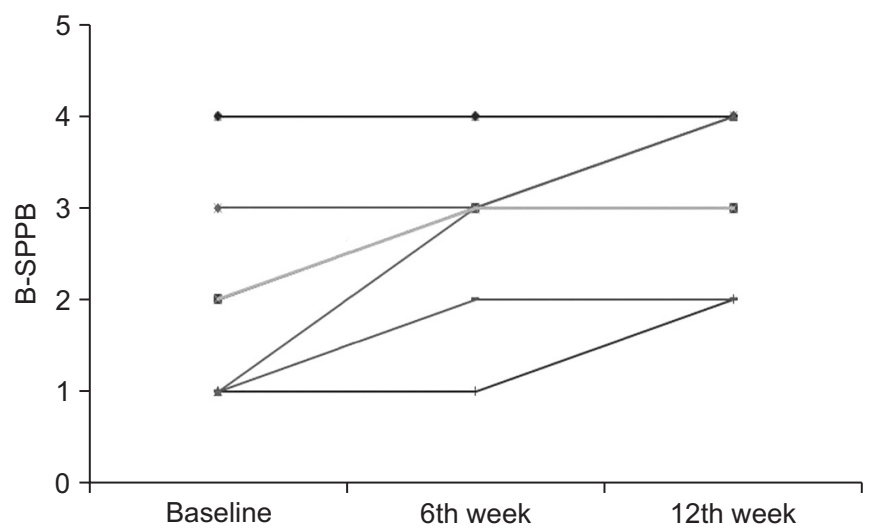

Fig. 5. Changes in the balance item of Short Physical Performance Battery (B-SPPB) for 13 patients in baseline, the 6 th week and 12 th week.

was observed for the remainder of the study. The other subjects completed the training without any kinds of side effects like severe pain and safety risks.

\section{Patients' satisfaction}

After completion of the training, the patients' satisfaction was evaluated with several questions. One of the questions was "Has your gait performance been improved after the training?" and the patients had to answer as from 1 to 5 points. One point meant 'definitely no' and 5 points meant 'definitely yes'. The mean score was 3.85 points. No one answered as 1 point, one patient answered as 2 points, four patients answered as 3 points, four patients answered as 4 points, and four patients answered as 5 points. Most patients felt there was improvement of gait function during and after the robot training with WA-H.
Table 5. Changes in the subscale of B-FMAS in baseline, the 6 th week and 12 th week $(n=13)$

\begin{tabular}{lcc}
\hline & B-FMAS & p-value \\
\hline Baseline & $10.5 \pm 1.5$ & \\
6th week & $10.9 \pm 1.3$ & 0.063 \\
12th week (end of training) & $11.6 \pm 1.3$ & $0.006^{*}$ \\
\hline
\end{tabular}

Values are presented as mean \pm standard deviation.

B-FMAS, balance function of Fugl-Meyer Assessment Scale.

${ }^{*} \mathrm{p}<0.05$ vs. baseline.

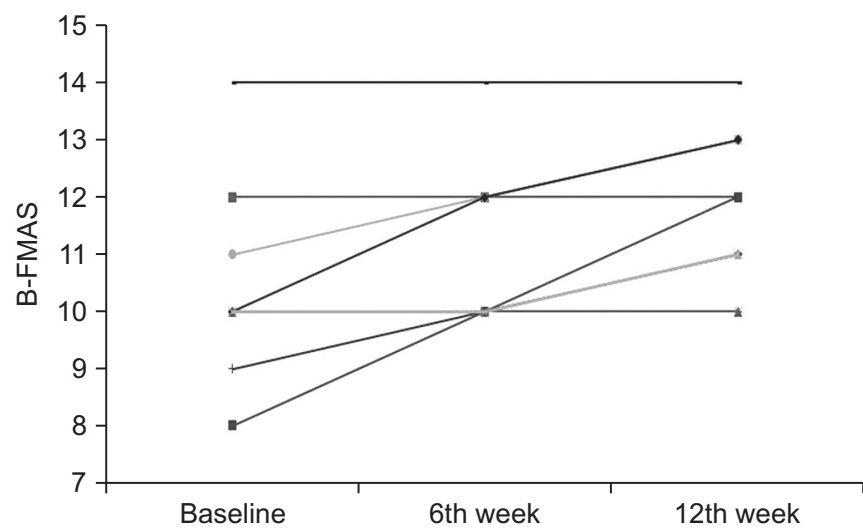

Fig. 6. Changes the subscale of balance function of FuglMeyer Assessment Scale (B-FMAS) for 13 patients in baseline, the 6 th week and 12th week.

\section{DISCUSSION}

The efficacy and feasibility of a rehabilitation program using the newly developed, portable and light-weight, wearable gait assistive robot, WA-H, was evaluated. This robot system was developed in HEXAR System, Seoul, Korea. WA-H is a portable and much less expensive exoskeletal robot developed for hemiplegic patients compared with Lokomat or ReWalk. This study is the first detailed study of the efficacy of WA-H for hemiplegics in Korea. There were no major or minor adverse results such as pain or equipment problems during training which might have interrupted the rehabilitation program. It was demonstrated that this wearable gait assistive robot using WA-H could be effectively and safely used for hemiplegic patients without the hard manual labor of physical therapists to improve gait parameters. In addition, significant improvements in multiple measurements of balance function were observed. Gait training with this wearable, gait assistive robot using WA-H could 
improve balance function and movement coordination in patients with neurological impairment [15]. These results showed improvement in all evaluation scales for balance function after 12 weeks of training thus demonstrating the feasibility of the newly developed, gait assistive robot for rehabilitation training.

Many recent studies on rehabilitation of hemiplegic patients using a wearable gait assistive robot have been published worldwide. Kubota et al. [3] used a Hybrid Assistive Limb (HAL; University of Tsukuba, Tsukuba, Japan) and reported significant improvement in gait speed, number of steps, and cadence after 8 weeks of rehabilitation, but showed no statistically significant improvement in balance scales like TUGT and BBS. Schwartz et al. [16] compared a rehabilitation group that used Lokomat with a group treated with conventional gait training, and reported statistically significant improvement in functional ambulatory capacity scores and the National Institutes of Health Stroke Scale (NIHSS). In contrast, Hidler et al. [17] reported statistically significant improvement in a conventional gait training group rather than a Lokomat group. Thus, there are many debates at present on the practicality and efficacy of rehabilitation training using wearable assistive robots.

Gait rehabilitation with this newly developed, gait assistive robot has the following advantages. Existing conventional training methods require various safety devices, space, two or more physical therapists for each patient, and equipment like treadmills, all of which are hindrances to accomplishing the training. Moreover, when considering the Korean medical system that requires a physical therapist to tend to many patients, therapists inevitably become fatigued during rehabilitation sessions. However, a robotic device such as WA-H has the advantage of providing rehabilitation in any place and at any time without barriers, so that it has a significant potential for further development. Moreover, since rehabilitation training using WA-H provides active and highly repetitive movements without fatigue, more intense and task-specific interventions are possible compared to a therapistdependent, repetitive, conventional rehabilitation. The robotic rehabilitation can show cortical reorganization and significant improvements in motor function [15]. Calabro et al. [18] reported that gait training with a robotic device increased patients' motor output, involvement, and motivation. This suggests that gait training with the newly developed, assistive robot would have potential to improve not only the practical balance function, but also the satisfaction and confidence of hemiplegic patients by improving stability and gait velocity. Further studies are needed to establish the psychological effect of gait training with the wearable, gait assistive robot.

In conclusion, gait training with the wearable, gait assistive robot, WA-H, seems to have a beneficial effect on balance function in patients with hemiplegia. WA-H was safe, and could be used clinically for hemiplegic gait training.

This study has certain limitations. First, this study was not a randomized controlled trial, and could not compare the efficacy of robotic training with conventional rehabilitation. An attempt was made to recruit a control group of subjects, but they did not want to participate in a control group which can do only evaluation sessions without any rewards like gait training with a robotic device. Thus a control group was not attainable. To assess clinical usefulness, it will be necessary to compare the present results with a control group who has been treated with conventional rehabilitation therapy for balance function. Future studies should assess a control group in order to confirm the clinical efficacy of training with the newly developed, gait assistive robot in comparison with conventional therapy. Second, this study could not exclude subject bias because all patients were recruited through a single rehabilitation facility. Third, the statistical power was low because of the small number of patients.

Wearable, gait assistive, robotic systems integrate the advanced technologies of mechanics, materials, electronics, bionics, control technology, and artificial intelligence [7]. To progress along with the continuously developing mechanical technology, the medical community should attempt to provide guidance in developing technologies related to medicine by cooperating with others so that such development would be helpful in treatment and rehabilitation of patients. There are many obstacles to overcome for a robotic technology to be clinically used safely. It must be light-weight, and have an excellent human-robot interface. Energy efficiency and cost are other factors that are important. Risk of fall must be minimized, and patient safety in the event of machinery error must be guaranteed. Also, most currently developed lower extremity exoskeletal robots are heavy, with limited torque and power. Powerful motors at hip and knee joints 
can apply regulated external force and torque to the patient's limbs and enhance the strength of patient's joints. Hemiplegic patients need sufficient external power to achieve gait movement. For the clinical use of the gait assistive robot for more critical patients and in outdoor environments, motors need a small volume, high powerto-weight ratio, high efficiency, and compliance [7]. Ideal timing and treatment protocols for robot-assisted rehabilitation must be also established for patients to proceed with rehabilitation without limitation of space and time. Further studies, including randomized controlled trials for comparison with conventional therapies, should be conducted with larger numbers of patients while considering disease progression and demographic factors of each patient. Integration of robotic therapy into current practice holds promise of improving the quality of physical rehabilitation by alleviating its labor-intensive aspects, and decreasing the costs related to therapists.

\section{CONFLICT OF INTEREST}

No potential conflict of interest relevant to this article was reported.

\section{ACKNOWLEDGMENTS}

This work was supported by Business for Cooperative $R \& D$ between Industry, Academy, and Research Institute funded Korea Small and Medium Business Administration in 2016 (Grant No. C0330956).

\section{REFERENCES}

1. Moseley AM, Stark A, Cameron ID, Pollock A. Treadmill training and body weight support for walking after stroke. Stroke 2003;34:3006.

2. Hiroyuki S, Uchiyama Y, Kakurai S. Specific effects of balance and gait exercises on physical function among the frail elderly. Clin Rehabil 2003;17:472-9.

3. Kubota S, Nakata Y, Eguchi K, Kawamoto H, Kamibayashi K, Sakane M, et al. Feasibility of rehabilitation training with a newly developed wearable robot for patients with limited mobility. Arch Phys Med Rehabil 2013;94:1080-7.

4. Lunenburger L, Colombo G, Riener R, Dietz V. Biofeedback in gait training with the robotic orthosis Lo- komat. Proceedings of the 26th Annual International Conference of the IEEE Engineering in Medicine and Biology Society (IEMBS); 2004 Sep 1-5; San Francisco, CA. p. 4888-91.

5. Neckel N, Wisman W, Hidler J. Limb alignment and kinematics inside a Lokomat robotic orthosis. Proceedings of the 28th Annual International Conference of the IEEE Engineering in Medicine and Biology Society (IEMBS); 2006 Aug 30-Sep 3; New York, NY. p. 2698-701.

6. Pohl M, Werner C, Holzgraefe M, Kroczek G, Mehrholz J, Wingendorf I, et al. Repetitive locomotor training and physiotherapy improve walking and basic activities of daily living after stroke: a single-blind, randomized multicentre trial (DEutsche GAngtrainerStudie, DEGAS). Clin Rehabil 2007;21:17-27.

7. Chen B, Ma H, Qin LY, Gao F, Chan KM, Law SW, et al. Recent developments and challenges of lower extremity exoskeletons. J Orthop Transl 2016;5:26-37.

8. Lee HD, Han CS. Technical trend of the lower limb exoskeleton system for the performance enhancement. J Inst Control Robot Syst 2014;20:364-71.

9. Ji YH, Yun DW, Jang HY, Lee DB, Khan AM, Kim S, et al. Study on efficacy of gait training for hemiplegia patients using lower-limb wearable robot. J Korean Soc Precis Eng 2015;32:879-83.

10. Oberg T, Karsznia A, Oberg K. Basic gait parameters: reference data for normal subjects, 10-79 years of age. J Rehabil Res Dev 1993;30:210-23.

11. Ng SS, Hui-Chan CW. The timed up \& go test: its reliability and association with lower-limb impairments and locomotor capacities in people with chronic stroke. Arch Phys Med Rehabil 2005;86:1641-7.

12. Fish J. Short physical performance battery. In: Kreutzer JS, DeLuca J, Caplan B, editors. Encyclopedia of clinical neuropsychology. New York: Springer; 2011. p. 2289-91.

13. Blum L, Korner-Bitensky N. Usefulness of the Berg Balance Scale in stroke rehabilitation: a systematic review. Phys Ther 2008;88:559-66.

14. Gladstone DJ, Danells CJ, Black SE. The Fugl-Meyer assessment of motor recovery after stroke: a critical review of its measurement properties. Neurorehabil Neural Repair 2002;16:232-40.

15. Sale P, Franceschini M, Waldner A, Hesse S. Use of the robot assisted gait therapy in rehabilitation of patients 
with stroke and spinal cord injury. Eur J Phys Rehabil Med 2012;48:111-21.

16. Schwartz I, Sajin A, Fisher I, Neeb M, Shochina M, Katz-Leurer M, et al. The effectiveness of locomotor therapy using robotic-assisted gait training in subacute stroke patients: a randomized controlled trial. PM R 2009;1:516-23.

17. Hidler J, Nichols D, Pelliccio M, Brady K, Campbell DD, Kahn JH, et al. Multicenter randomized clinical trial evaluating the effectiveness of the Lokomat in subacute stroke. Neurorehabil Neural Repair 2009;23: 5-13.

18. Calabro RS, Reitano S, Leo A, De Luca R, Melegari C, Bramanti $\mathrm{P}$. Can robot-assisted movement training (Lokomat) improve functional recovery and psychological well-being in chronic stroke? Promising findings from a case study. Funct Neurol 2014;29:139-41. 